\title{
A Theoretical Model of a Molecular-Motor-Powered Pump
}

\author{
Joseph L. Bull,, ${ }^{1, *}$ Alan J. Hunt, ${ }^{1}$ and Edgar Meyhöfer ${ }^{2}$ \\ ${ }^{1}$ Department of Biomedical Engineering, \\ ${ }^{2}$ Department of Mechanical Engineering, The University of Michigan, \\ Ann Arbor, MI 48109, USA E-mail: joebull@umich.edu
}

\begin{abstract}
The motion of a cylindrical bead in a fluid contained within a two-dimensional channel is investigated using the boundary element method as a model of a biomolecular-motor-powered microfluidics pump. The novelty of the pump lies in the use of motor proteins (kinesin) to power the bead motion and the few moving parts comprising the pump. The performance and feasibility of this pump design is investigated using two model geometries: a straight channel, and a curved channel with two concentric circular walls. In the straight channel geometry, it is shown that increasing the bead radius relative to the channel width, increases the flow rate at the expense of increasing the force the kinesins must generate in order to move the bead. Pump efficiency is generally higher for larger bead radii, and larger beads can support higher imposed loads. In the circular channel geometry, it is shown that bead rotation modifies the force required to move the bead and that shifting the bead inward slightly reduces the required force. Bead rotation has a minimal effect on flow rate. Recirculation regions, which can develop between the bead and the channel walls, influence the stresses and force on the bead. These results suggest this pump design is feasible, and the kinesin molecules provide sufficient force to deliver pico- to atto- $1 / \mathrm{s}$ flows.
\end{abstract}

Key Words. microfluidics, kinesin, Stokes flow, boundary element method

\section{Introduction}

Microfluidics circuits often do not include a pump and flow must be generated using either gravity or an external, macro-scale pump. Simply scaling down a conventional pump does not provide the desired performance. This is due in part to many conventional pumps being designed to work at high Reynolds numbers, $R e=\rho U a / \mu$, i.e. the ratio of inertial to viscous effects is high, where $U$ is the characteristic velocity scale, $a$ is the characteristic length scale, $\rho$ is fluid density, and $\mu$ is fluid viscosity. In many microfluidic devices, $R e$ is small due to the small length scales. Thus, many micro-flows lack the inertia required for efficient function of these pump designs. Additionally, conventional pumps often have intricate designs that are difficult to manufacture at the micro-scale. Besides macroscale syringe pumps, a number of novel pump designs have used a variety of driving mechanisms, such as expanding and collapsing bubbles, electro-convection, surface tension, and gravity (Yuan and Prosperetti, 1999; Ory et al., 2000, Geng et al., 2001; Ateya et al., 2004; Li et al., 2004; Min et al., 2004). While these designs have been success- ful in generating flow, most of these require relatively large external equipment, or require the microfluidics circuit to be positioned in a certain orientation. In ongoing work, we are interested in developing a molecular-motor-powered microfluidics pump that is truly micro-scale, robust, and highly efficient.

Kinesins are molecular motors that move along microtubules forming an intracellular transport system that is responsible for transporting vesicles and organelles between locations within the cell (Howard, 1996; Hirokawa, 1998). The two heads of these motor proteins provide the motor function, and the tail of the protein provides an attachment point for the payload. An individual kinesin will translocate along microtubles at a relatively constant rate (Hunt et al., 1994; Meyhofer and Howard, 1995) over a wide range of imposed load, and each kinesin can generate a force on the order of $10 \mathrm{pN}$ (Hunt et al., 1994; Meyhofer and Howard, 1995). They are small (head domain $\sim 7 \mathrm{~nm}$, overall $\sim 50 \mathrm{~nm}$ ), robust, and possess a high thermodynamic efficiency $(\sim 50-60 \%)$ and a high power to mass ratio $(4400 \mathrm{~W} / \mathrm{kg})$. By comparison, the typical car engine has a thermodynamic efficiency $\sim 30 \%$ and a power to mass ratio of $\sim 300 \mathrm{~W} / \mathrm{kg}$. The efficiency, size and power density of kinesin suggest it may be an appropriate power source for sophisticated microdevices, and a number of reports in the literature have investigated the potential use of kinesin in microdevices (Limberis and Stewart, 2000; Moorjani et al., 2003; Hess et al., 2004; Jia et al., 2004). We are developing a potential device for flow generation that consists of a kinesin- (or microtubule-) coated bead or cylinder moving along a microtubule (kinesin) track in a channel.

To investigate the feasibility of this micropump design, we consider a two-dimensional model in which a cylinder moves with prescribed translational and rotational speeds through a channel. Flow past cylinders in a free stream has been studied in many contexts, such as heat exchangers and membrane oxygenators (Sparrow and Loeffler, 1959; Wang, 1996; Dierickx et al., 2000). Other studies have considered the flow induced by the motion of spheres (Dean and O'Neill, 1963; O'Neill, 1964). Flow around cylinders and spheres inside channels and near

${ }^{*}$ Corresponding author. 
boundaries has been studied in many applications, such as transport of particles in sedimentation, and transport of red blood cells in capillaries (Dvinsky and Popel, 1987a, 1987b; Sugiharaseki and Skalak, 1988; Halpern and Secomb, 1991, 1992; Damiano et al., 1996, 2004; Feng et al., 1998), as well as for primarily fundamental interests (Bretherton 1962, Goldman et al., 1967a, 1967b; Goren and Oneill 1971; Ganatos et al., 1978, 1980a, 1980b, 1982; Larson and Higdon, 1986, 1987; Eklund and Jernqvist, 1994; Pozrikidis, 1994; SugiharaSeki, 1996; Staben et al., 2003). Most of these previous studies have considered objects that are held stationary as fluid flows around them, or objects that are carried along by the flow. There is much less work on flow produced by objects moving in channels. Most work in this area has been motivated by fundamental interest in particles moving in closed tubes and channels (Happel and Brenner, 1965; Davis, 1992), or by the needle viscometer (Thiessen and Krantz, 1992; Wehbeh et al., 1993; Liu et al., 2004), which primarily considers long cylinders translating in closed tubes. The current theoretical study investigates the efficiency of this pumping mechanism, the potential to generate useful flow rates, the effects of bead rotation and position, and the effects of channel curvature.

\section{Model}

Although we expect that the actual pump will involve a series of micro-scale, check valves (Hasselbrink et al., 2002; Kirby et al., 2002), we ignore the effects of these valves in this preliminary investigation. Two model geometries are considered - a straight channel and a circular channel model. The actual pump might consist of the circular channel connected to an external circuit.

\subsection{Straight channel}

First, we consider the limit in which channel curvature is negligible and use the simplified model geometry of a straight two-dimensional channel with periodic boundary conditions at the ends of the channel as shown in Figure 1. By specifying a pressure drop, $\Delta p^{*}$, across the ends of the channel, we approximate an applied loading on the pump. The channel half-width and length are $a$ and $L$, respec-

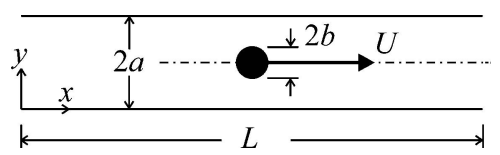

Fig. 1. Straight channel model geometry. The two-dimensional, circular bead of radius $b$ translates with constant speed $U$ through the channel of half-width a. tively, and a single bead of radius $b$ translates along the channel at a constant speed $U$. We use the notation that * superscripts indicate dimensional variables. Dimensionless variables and dimensional parameters are un-starred. The channel is filled with a Newtonian, incompressible liquid. The governing fluid mechanical equations are conservation of momentum

$$
\begin{aligned}
& \rho\left(\frac{\partial \vec{u}^{*}}{\partial t^{*}}+\left(\vec{u}^{*} \cdot \nabla^{*}\right) \vec{u}^{*}\right) \\
& \quad=-\nabla^{*} p^{*}+\mu \nabla^{* 2}+\rho \vec{g}
\end{aligned}
$$

and conservation of mass

$$
\nabla^{*} \cdot \vec{u}^{*}=0
$$

where $\vec{x}^{*}=x^{*} \hat{i}+y^{*} \hat{j}$ is position, $\vec{u}^{*}=u^{*} \hat{i}+v^{*} \hat{j}$ is velocity, $p^{*}$ is pressure, and $\hat{i}$ and $\hat{j}$ are unit vectors in the $x^{*}$ and $y^{*}$ directions, respectively. The boundary conditions are no slip and no penetration at the channel walls

$$
\begin{aligned}
& \vec{u}^{*}\left(x^{*}, y^{*}=0\right)=0 \\
& \vec{u}^{*}\left(x^{*}, y^{*}=2 a\right)=0
\end{aligned}
$$

and the imposed pressure difference at the ends of the channel

$$
\begin{aligned}
& p^{*}\left(x^{*}=0\right)=0 \\
& p^{*}\left(x^{*}=L\right)=\Delta p^{*}
\end{aligned}
$$

In the straight channel model, the bead translates along the channel centerline $\left(y^{*}=a\right)$ but does not rotate. The boundary conditions at the bead surface are

$$
\begin{aligned}
& u_{\text {bead }}^{*}=U \\
& v_{\text {bead }}^{*}=0
\end{aligned}
$$

The governing equations and boundary conditions are non-dimensionalized by the following scales:

$$
\begin{aligned}
& \vec{x}^{*}=a \vec{x} \\
& \vec{u}^{*}=U \vec{u} \\
& p^{*}=\mu U p / a
\end{aligned}
$$

where $\vec{x}, \vec{u}$ and $p$ are the dimesionless position, velocity and pressure, respectively. Note that for two-dimensional Poiseuille flow in a channel with half-width $a$, the pressure drop in a distance $a / 2$ along the channel is given by $\mu$. $u_{\text {max }} / a$, where $u_{\max }$ is the maximum velocity. We consider the limit of negligible gravitational effects and $R e \rightarrow 0$, as is the case in the device due to the small dimensions of the channel. The dimensionless governing equations are conservation of fluid mass and the Stokes equation.

$$
\begin{aligned}
\nabla \cdot \vec{u} & =0 \\
-\nabla p+\nabla^{2} \vec{u} & =0
\end{aligned}
$$




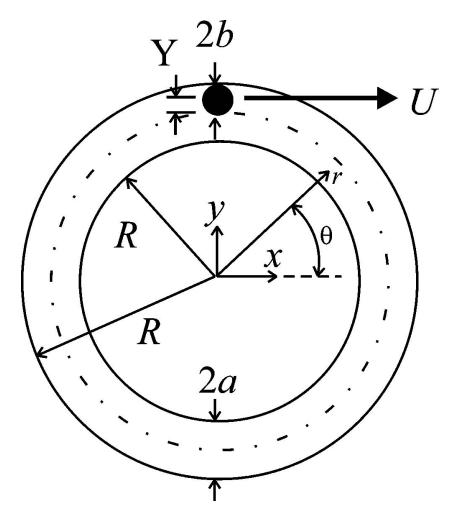

Fig. 2. Circular channel model geometry. The bead translates along the channel bounded by two concentric circular walls. The bead may rotate with prescribed angular velocity $\omega$ as it translates with speed $U$, and may be shifted from the centerline of the channel by a distance $Y$.

These governing equations are solved using the boundary element method, as described below. The effect of bead radius $(b / a)$, channel length $(L / a)$, and applied pressure drop $(\Delta p)$ on resulting flow $(Q)$ and the force $\left(F_{x}\right)$ required to move the bead were investigated.

\subsection{Curved channel}

The second geometry is a circular channel, as shown in Figure 2. As in the straight channel model, the channel half-width is $a$, but now the outer radius of the circular channel is $R_{o}$, and the inner radius is $R_{i}$. The origin of the polar coordinate system $\left(r^{*}, \theta\right)$ is at the center of the concentric inner and outer walls. In addition to channel curvature, we investigate the effects of bead shift, $Y$, from the centerline of the channel and bead rotation, $\omega$, in this more complicated geometry. No slip and no penetration boundary conditions are imposed at the channel wall, and the fluid velocity is required to equal the bead velocity at the bead surface. The governing equations are the same in this model geometry as in the straight channel, and they are non-dimensionalized using the same scales.

\subsection{Solution method}

The boundary element method (Brebbia and Dominguez, 1992; Pozrikidis, 1992) is used to solve the governing equations and compute the resulting velocity and pressure fields. A brief description follows. The solution for the velocity field resulting from Stokes flow can be obtained in terms of single and double layer potentials by taking Fourier transforms of equation (1) and applying Green's theorem (Ladyzhenskaya, 1963; Pozrikidis, 1992). This results in the following integral equation that must be sat- isfied by the flow.

$$
\begin{aligned}
u_{k}\left(\vec{x}_{0}\right)= & -\frac{1}{4 \pi} \int_{C} f_{i}(\vec{x}) G_{i j}\left(\vec{x}, \vec{x}_{0}\right) d l(\vec{x}) \\
& +\frac{1}{4 \pi} \int_{C} u_{i}(\vec{x}) T_{i j k}\left(\vec{x}, \vec{x}_{0}\right) n_{k}(\vec{x}) d l(\vec{x})
\end{aligned}
$$

As $\vec{x}_{0}$ approaches the boundary, equation (8) becomes

$$
\begin{aligned}
c_{k j} u_{k}\left(\vec{x}_{0}\right)= & -\frac{1}{4 \pi} \int_{C} f_{i}\left(\vec{x}, \vec{x}_{0}\right) G_{i j}\left(\vec{x}, \vec{x}_{0}\right) d l(\vec{x}) \\
& +\frac{1}{4 \pi} \int_{C} u_{i}(\vec{x}) T_{i j k}\left(\vec{x}, \vec{x}_{0}\right) n_{k}(\vec{x}) d l(\vec{x})
\end{aligned}
$$

where the curve $C$ is the selected flow boundary; $\vec{f}=\underline{\underline{\sigma}} \cdot \hat{n}$, is the stress vector; $\hat{n}$ is the unit normal pointing into the fluid; $\underline{\underline{\sigma}}=\left(-p \underline{\underline{I}}+\nabla \vec{u}+[\nabla \vec{u}]^{T}\right)$ is the stress tensor; and the two-dimensional Stokeslet and associated stress field are given respectively by

$$
\begin{aligned}
G_{i j} & =-\delta_{i j} \ln \left|\vec{x}-\vec{x}_{0}\right|+\frac{\left(x_{i}-x_{0 i}\right)\left(x_{j}-x_{0 j}\right)}{\left(\vec{x}-\vec{x}_{0}\right)^{2}} \\
T_{i j k} & =-4 \frac{\left(x_{i}-x_{0 i}\right)\left(x_{j}-x_{0 j}\right)\left(x_{k}-x_{0 k}\right)}{\left(\vec{x}-\vec{x}_{0}\right)^{4}}
\end{aligned}
$$

The tensor $c_{k j}$ is due to the stress jump at the boundaries. For smooth surfaces it has the value $\delta_{k j} / 2$, but is more complicated if the domain has corners. The subscripts refer to direction, i.e. $x_{1}=x$ and $x_{2}=y$. Equation (9) is solved numerically by discretizing the boundary into $N$ quadratic elements. The velocity and stress vectors, $\vec{u}$ and $\vec{f}$, are discretized along the boundary and represented by quadratic polynomials. The governing integral equation then becomes

$$
\begin{aligned}
c_{k j} u_{k}\left(\vec{x}_{0}\right)= & -\frac{1}{4 \pi} \sum_{n=1}^{N} \int_{C_{n}} f_{i}(\vec{x}) G_{i j}\left(\vec{x}, \vec{x}_{0}\right) d l(\vec{x}) \\
& +\frac{1}{4 \pi} \sum_{n=1}^{N} \int_{C_{n}} u_{i}(\vec{x}) T_{i j k}\left(\vec{x}, \vec{x}_{0}\right) \\
& \times n_{k}(\vec{x}) d l(\vec{x})
\end{aligned}
$$

where $n$ indicates the element number and $C_{n}$ refers to the contour along element $n$. Equation (11) can be represented by a system of linear equations,

$$
\underline{\underline{\hat{H}}} \underline{w}=\underline{\underline{\hat{G}}} \underline{t}
$$

where $\underline{\underline{\hat{H}}}$ and $\underline{\underline{G}}$ are $4 N \times 4 N$ and $4 N \times 6 N$ matrices, respectively. The vectors $\underline{w}$ and $\underline{t}$ have lengths of $4 N$ and 
$6 N$, respectively, and $w_{2 n-1}=u_{n}, w_{2 n}=v_{n}, t_{2 n-1}=f_{x n}$, and $t_{2 n}=f_{y n}$.

The elements of $\underline{\underline{\hat{H}}}$ and $\underline{\underline{\hat{G}}}$ are computed using a 10point Gaussian quadrature if $\overrightarrow{\vec{x}}$ doesn't coincide with one of the node points of $C_{n}$. A 10-point logarithmic quadrature is used to evaluate the sections of the integral on which $\vec{x}$ does coincide with a node point.

We impose two boundary conditions (selected from $u$, $v, f_{x}$ and $f_{y}$ ) at each node point, and rearrange equation (12) to obtain

$$
\underline{\underline{A}} \underline{z}=\underline{b}
$$

where $\underline{A}$ is a $4 N \times 4 N$ matrix, $z$ is a $4 N$ vector containing the unknown stresses and velocities, and $\underline{b}$ is a $4 N$ vector that contains the prescribed boundary condition information. Equation (13) is then solved, using Gaussian elimination with partial pivoting, for the two unknown boundary values at each node point. Once all components of stress and velocity are known at the boundary nodes, the velocity and stress can be determined at any interior point, e.g. equation (8). A powerful feature of the boundary element method is that only the boundary must be discretized in order to solve for the unknowns on the boundary. This facilitates the analysis of flows within complicated geometries, allows the consideration of deforming and moving boundaries without re-meshing of the boundary nodes at each time step, and results in relatively fast computation times.

\section{Results and discussion}

A range of dimensionless parameter values, corresponding to values of the micropump, was considered. We investigated the effects of bead diameter, $b / a$, and channel length, $L / a$, on the flow, $Q$, generated, subject to the speed and available power of the kinesin motors. Typical kinesin speeds are $U=0.8$ to $1 \mu \mathrm{m} / \mathrm{s}$, and depend on the type of kinesin. Additionally, for the curved channel model, the effects of bead shift, $Y / a$, from the channel centerline and bead rotation, $\omega$, were investigated. The force, $F_{x}$, per dimensionless unit depth normal to the plane of the two-dimensional model required to move the bead was calculated by integrating the stress over the bead surface,

$$
F_{x}=\int_{\text {bead }} \vec{f} \cdot \hat{i} d l
$$

using a trapezoid rule approximation for the integral. Note that dimensional force $F_{x}^{*}=\mu U a F_{x}$. Likewise the dimensionless flow rate per unit depth into the page, $Q$, generated by the bead motion was determined by numerically integrating the velocity over the channel cross-section:

$$
Q=\int_{y=0}^{y=2} \vec{u} \cdot \hat{i} d y
$$

for the straight channel or

$$
Q=\int_{r=R_{i} / a}^{r=R_{o} / a} \vec{u} \cdot \hat{n} d r
$$

for the curved channel.

The numerical solution the model was conducted on a 4-processor Sun Fire V880 equipped with 8 GB of RAM (Sun Microsystems, Santa Clara, CA). The convergence of the code was investigated for both the straight channel and the curved channel models. Both geometries resulted in convergence for a relative small number of node points. For simplicity, 640 node points were used along the channel walls and 160 node points on the bead surface in each case. For the calculation of the interior node points, $\sim 2000$ node points (the exact number depended on which geometry was used) were placed evenly, in $(x, y)$ for the straight channel or $(r, \theta)$ for the circular channel, to form a grid on which the velocity and pressure fields were calculated. This resulted in convergence (for $F_{x}, Q$, pressure, and streamlines) for all of the conditions investigated. The interior node points were only constructed if the streamlines were computed. When they were not included, the code required $\leq 1$ minute to run. If they were included, the computation time required was typically $\leq 30 \mathrm{~min}-$ utes. Note that only the boundary nodes were needed to calculate $F_{x}$ and $Q$ in the straight channel geometry, and that only a few internal node points were required in addition to the boundary nodes to calculate the $Q$ (and none for $F_{x}$ ) in the circular channel geometry. This results in fast computation speeds and could potentially facilitate a rapid optimization of the pump geometry based only on minimizing $F_{x}$ and maximizing $Q$. The speed and adaptability of the boundary element method suggest it is a powerful and versatile method for investigating flows and performance of molecular-motor-driven microfluidic devices, such as this pump. The internal points were needed to determine the velocity and stress fields in the liquid, and significantly increased the computational time, which was still fast compared to other computational methods. As an additional check on accuracy, the straight channel model was modified to consider flow past a stationary cylinder and the resulting force and torque on the cylinder were compared to the results of previous studies (Dvinsky and Popel, 1987a, 1987b) with excellent agreement. The results of our study of flow induced by cylinder motion in the setting of a kinesin-powered micropump are described below. 


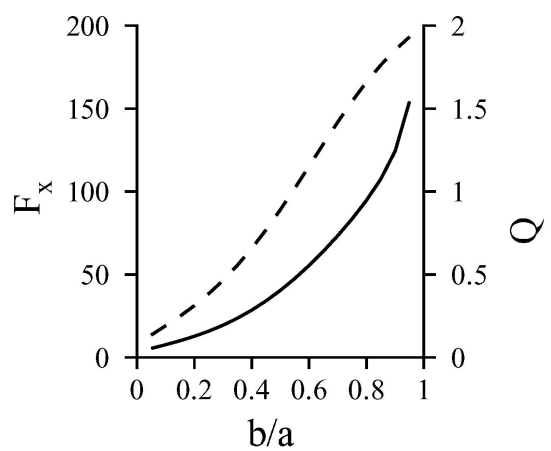

Fig. 3. Dimensionless force, $F_{x},--$, and dimensionless flow rate, $Q$, ,$-- v s$. dimensionless bead radius, b/a, in the straight channel with $L / a=20$ and $\Delta p=0$.

\subsection{Straight channel}

As shown in Figure 3, we computed the dimensionless force required to move the bead, $F_{x}$, and the resulting dimensionless flow rate, $Q$, for various values of the dimensionless bead radius, $b / a$. As the bead radius is increased, $Q$ is increased, but at the expense of increasing $F_{x}$. As $b / a$ approaches $1, Q$ approaches 2, which is the value corresponding to all of the fluid in the channel moving with the same velocity as the bead. The data shown in Figure 3 correspond to $L / a=20$ and $\Delta p=0 . F_{x}$ increases with $b / a$, partly because the increase in $Q$ results in an increase in pressure drop along the channel due to the Poisuielle flow in the region away from the bead and partly because of increased resistance in the region near the bead as fluid is squeezed between the bead and the wall. As the liquid film separating the bead from the wall becomes very thin, the viscous resistance increases significantly, accounting for the sharp increase in $F_{x}$ near $b / a=0.9$. Assuming 10 kinesin are in contact with the microtubule track, the available dimensional force to move the bead is $100 \mathrm{pN}$. Converting this dimensional force, $F_{x}^{*}=\mu \cdot U \cdot a \cdot F_{x}$, to a dimensionless force yields $F_{x}=5000$ to 500,000 , corresponding to $a=10 \mathrm{~nm}$ to $10 \mu \mathrm{m}, U \approx 1 \mu \mathrm{m} / \mathrm{s}$, and $\mu \approx 1 \times 10^{-3} \mathrm{~N} \cdot \mathrm{s} / \mathrm{m}^{2}$. This is much greater than the value of $F_{x}(<200$ as shown in Figure 3 ) required to move the bead, suggesting the available kinesin will provide sufficient force to power the pump. Dimensionally, the required force and resulting flow are approximately $1.5 \mathrm{pN}$ and 0.2 $\mathrm{pl} / \mathrm{s}$, respectively, for $a=10 \mu \mathrm{m}, L=200 \mu \mathrm{m}, U \approx 1$ $\mu \mathrm{m} / \mathrm{s}$, and $\mu \approx 1 \times 10^{-3} \mathrm{~N} \cdot \mathrm{s} / \mathrm{m}^{2}$.

The force, $F_{x}$, required to move the bead vs. channel length, $L / a$, and $Q$ vs. $L / a$ are plotted in Figure 4(a) and (b), respectively, for $b / a=0.1,0.5$, and 0.9 . The relationship between $F_{x}$ and $L / a$ is nearly linear for $b / a=0.9$ for all values of $L / a$, and is nearly linear for $b / a=0.1$ and $b / a=0.5$ when $L / a$ is greater than approximately 20. As $b / a \rightarrow 1$ (i.e. the bead fills most of the channel) there is little leakage of fluid around the bead and the ve-

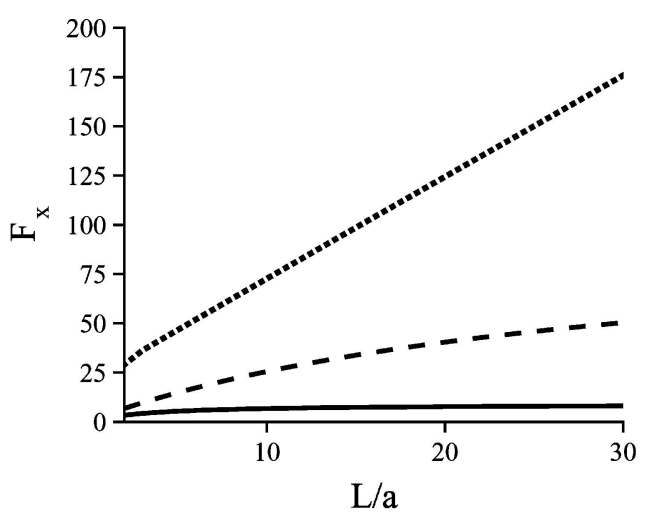

(a)

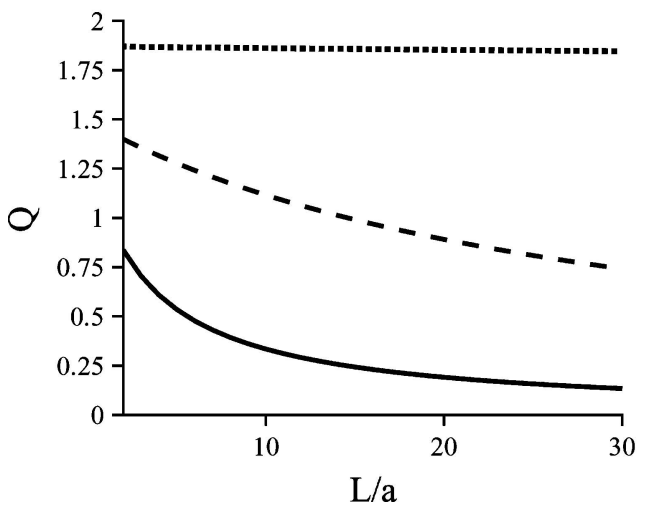

(b)

Fig. 4. (a) Dimensionless force, $F_{x}$, vs. dimensionless channel length, $L / a$, and $(b)$ dimensionless flow rate, $Q$, vs. dimensionless channel length, L/a. Each plot contains lines corresponding to $b / a=0.1,--$, $b / a=0.5,--$, and $b / a=0.9, \ldots$. These data are from the straight channel model.

locity profile is expected to be parabolic (as in Poiseuille flow) away from the bead. If the only contribution to Fx were the viscous losses due to Poiseuille flow in the region away from the bead, one would expect $F_{x} \rightarrow 0$ as $L / a \rightarrow$ 0 (note $1 \leq L / a \leq 30$ in Figure 4). However, this is not the case, indicating the flow field near the bead contributes significantly to $F_{x}$.

A simplistic approach of considering an applied load to the pump was to impose a pressure drop, $\Delta p$, across the ends of the channel, with the assumptions that one-way micro-valves could be used to connect the pump to an external circuit and that a constant $\Delta p$ will provide some insights into the behavior of the pump under those conditions. The flow details of such micro-valves are neglected, and it is noted that members of our group have previously constructed such valves (Hasselbrink et al., 2002; Kirby et al., 2002). The mechanical efficiency of the pump, $\eta$, is defined as the power delivered to the fluid $\left(Q^{*} \Delta p^{*}\right)$ divided by the power input to the pump $\left(F_{x}^{*} U\right)$. The efficiency, $\eta$, is dimensionless, and non-dimensionalizing the remaining terms, results in

$$
\eta=Q \Delta p / F_{x}
$$



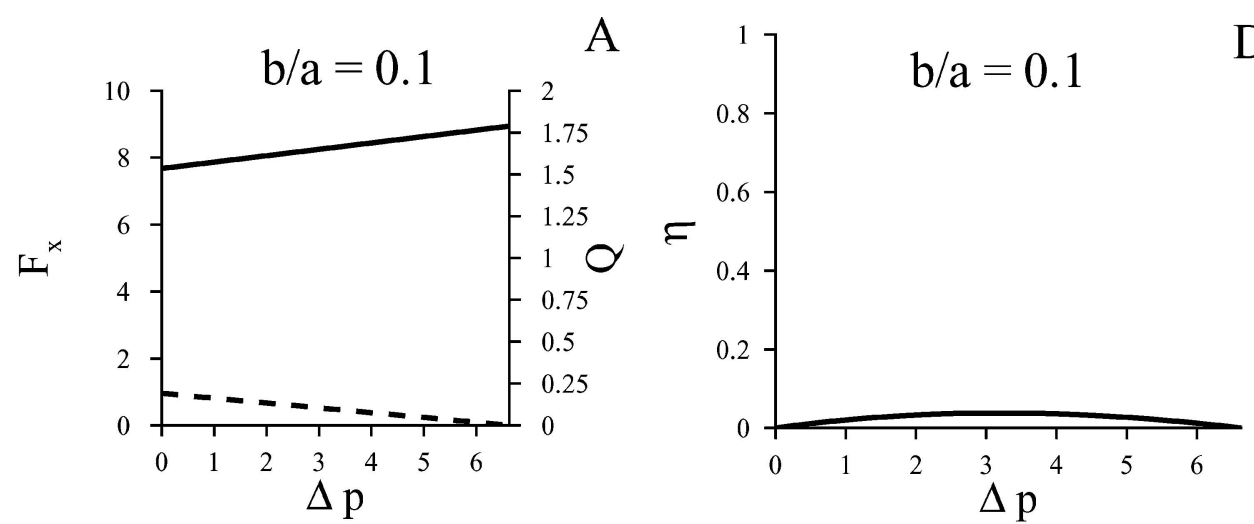

B
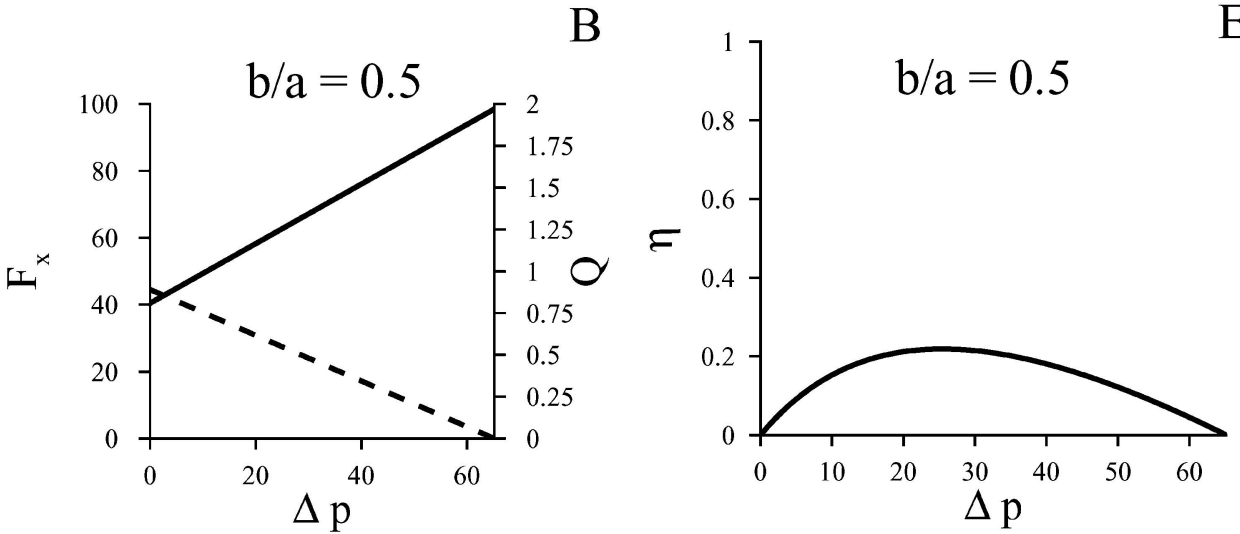

$\mathrm{E}$

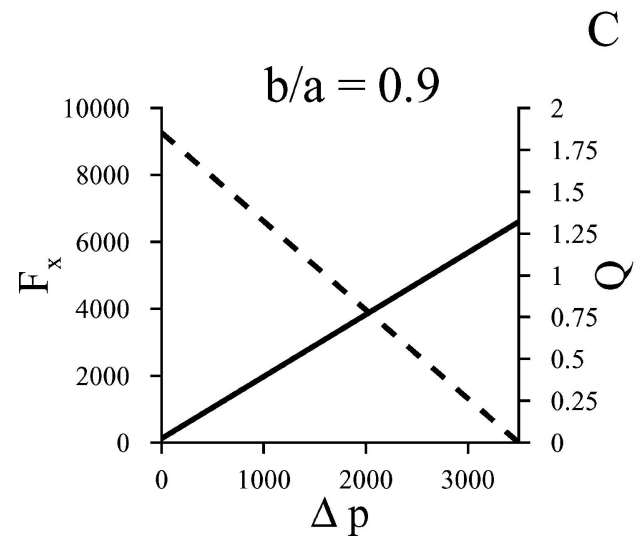

$\mathrm{C}$

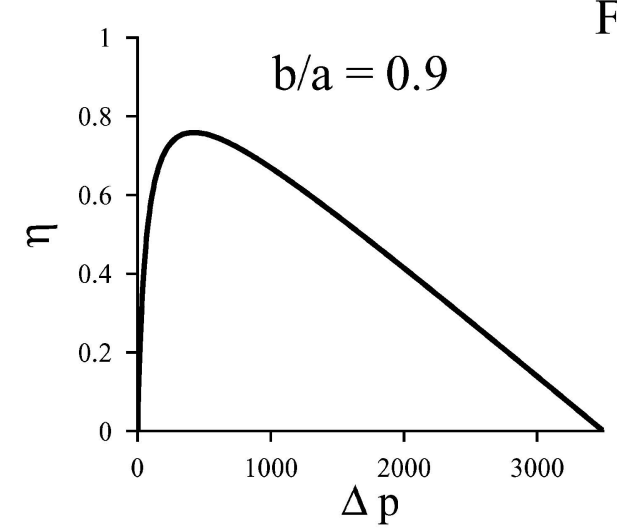

Fig. 5. Force, $F_{x}$,--, and flow rate, $Q$, --, vs. imposed loading, $\Delta p$ for (a) b/a $=0.1,(b) b / a=0.5$, and (c) b/a $=0.9$. Efficiency, $\eta$, vs. $\Delta p$, for (d) $b / a=0.1,(e) b / a=0.5$, and $(f) b / a=0.9$. These data are from the straight channel model with $L / a=20 . \Delta p$ represents the load applied to the pump.

Figure 5 contains plots of $F_{x}$ and $Q$ vs. $\Delta p$ for (a) $b / a$ $=0.1$, (b) $b / a=0.5$ and (c) $b / a=0.9$, as well as plots of efficiency, $\eta$, vs. $\Delta p$ for (d) $b / a=0.1$, (e) $b / a=0.5$ and (f) $b / a=0.9$. In these plots, $L / a=20$. The pump efficiency is higher for the larger value of $b / a$. This is partly because the when $b / a$ is large there is less leakage of fluid around the bead, and, consequently, a higher $\Delta p$ can be supported before $Q$ becomes negative. For each value of $b / a, \eta$ has a local maximum, corresponding to an optimal $\Delta p$ at which the pump is most efficient. For this model, which neglects the effects of valves, $\eta$ can exceed $80 \%$
(Figure 5) and depends on the bead size and $\Delta p$. Theoretical predictions of mechanical efficiencies for flagellar propulsion yield $\eta_{\max } \approx 3 \%$ (Childress 1981), indicating the model pump could potentially be very efficient for this size scale. Smaller beads result in lower efficiencies, because the bead motion does not induce much flow, but the bead still must overcome the viscous resistance opposing its motion.

The fluid pressure, $p$, at the centerline of the channel vs. position, $x$; the $x$ component of wall stress, $f_{x}$, vs. $x$; and the $y$-component of wall stress, $f_{y}$, vs. $x$ are plotted in 


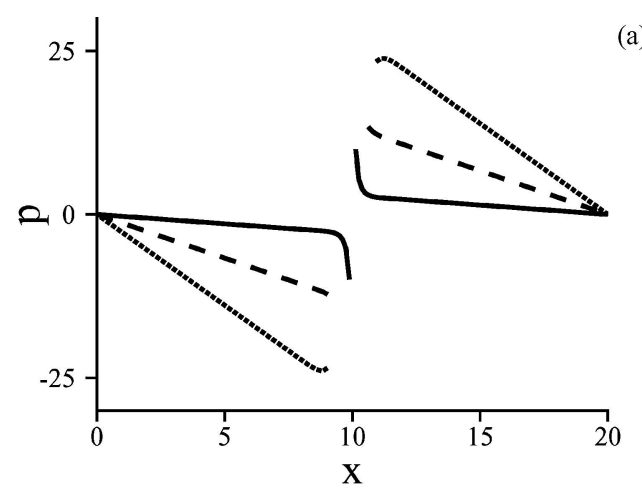

(a)

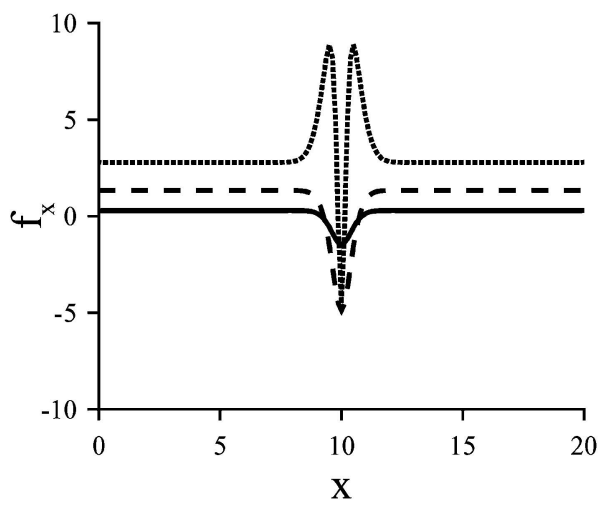

(b)

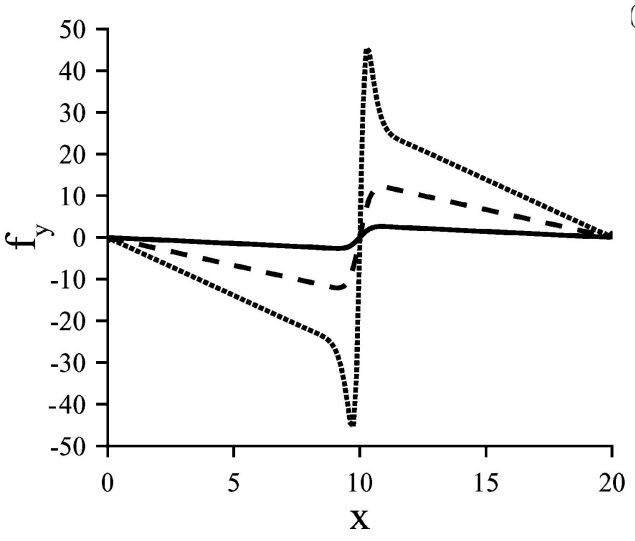

Fig. 6. (a) Channel centerline pressure, $p(y=0)$, vs. longitudinal position, $x$, (b) $x$-component of wall stress, $f_{x}(y=1)$, vs. $x$, and $(c)$ $y$-component of wall stress, $f_{y}(y=1)$, vs. $x$. Each plot contains lines corresponding to $b / a=0.1,--, b / a=0.5,--$, and $b / a=0.9, \ldots$. These data are from the straight channel model. Note that in (a) there is a jump in the pressure curves at the bead because the centerline pressure within the bead is undefined.

Figure 6(a)-(c), respectively, for $b / a=0.1,0.5$, and 0.9 . Stresses and pressure increase as the bead radius increases. At locations away from the bead ( $x$ not near 10), the longitudinal pressure gradient, $\partial p / \partial x$, is nearly constant, $f_{y}$ is linear in $x$, and $f_{x}$ is constant, as would be expected in Poiseuille flow. Note that Poiseuille flow has a parabolic velocity profile and is the typical fully-developed laminar flow in two-dimensional channels or cylindrical tubes
(Sutera and Skalak 1993; Pozrikidis, 1997a). For a twodimensional channel of half-width $a$, the relationship between the flow rate (per unit depth) and the constant pressure gradient, in dimensional terms is

$$
Q^{*}=-\frac{2 a^{3}}{3 \mu} \frac{\partial p^{*}}{\partial x^{*}}
$$

Non-dimensionlizing equation (18) by the previously described characteristic scales and rearranging yields

$$
\frac{\partial p}{\partial x}=-\frac{3}{2} Q
$$

The slopes of the lines in Figure 6(a) are well approximated by equation (19) away from the bead $(x=10)$. However, near the bead, there are sudden changes in $p$, $f_{x}$, and $f_{y}$, indicating the flow in the vicinity of the bead is not Poiseuille flow. There is a jump in pressure across the bead, with high pressure ahead of the bead and low pressure behind the bead to induce motion of the fluid. The value of $f_{x}$ along the wall is negative near the bead center $(x=10)$ suggesting recirculation between the wall and bead (as demonstrated in Figure 7). For $b / a=0.9, f_{x}$ increases near the front and rear of the bead $(x=10 \pm b / a)$, and becomes negative near $x=10$, due to the very thin gap between the wall and bead that any fluid not swept along by the bead must squeeze through.

Figure 7 shows the streamlines in the region $0 \leq y \leq 1$ when $b / a x=0.95, L / a=20$. Away from the bead, the streamlines are parallel to the channel walls, and the velocity field has a parabolic shape corresponding to Poiseuille flow. Near the bead, the flow is not uni-directional and the bead affects the velocity field. A small recirculation region develops between the bead and the channel wall, as shown in Figure 7. For larger beads, the force required to move the bead is not simply a result of Poiseuille flow, but is significantly influenced by the flow near the bead, as indicated by the large pressure gradients near the bead. The close spacing of the pressure contours near $x=10$ in Figure 7(c) corresponds to a higher pressure gradient than in (a) and (b).

\subsection{Curved channel}

Figure 8 shows (a) dimensionless force, $F_{x}$, and (b) dimensionless flow rate, $Q$, vs. dimensionless bead radius, $b / a$, for $R_{o} / a=3$ and $R_{o} / a=10$. These two values of $R_{o} / a$ were selected to allow investigation of channel curvature effects. Both sizes could be used for the pump, as well as less curved channels. As in the straight channel geometry (Figure 3), increasing $b / a$ increases both $F_{x}$ and $Q$, and $Q$ approaches $Q_{\max }=2$ as $b / a$ approaches 1 . At a given value of $b / a, R_{o} / a=10$ results in a higher $F_{x}$ and lower values of $Q$ for $0<b / a<0.95$ compared to $R_{o} / a=3$, 


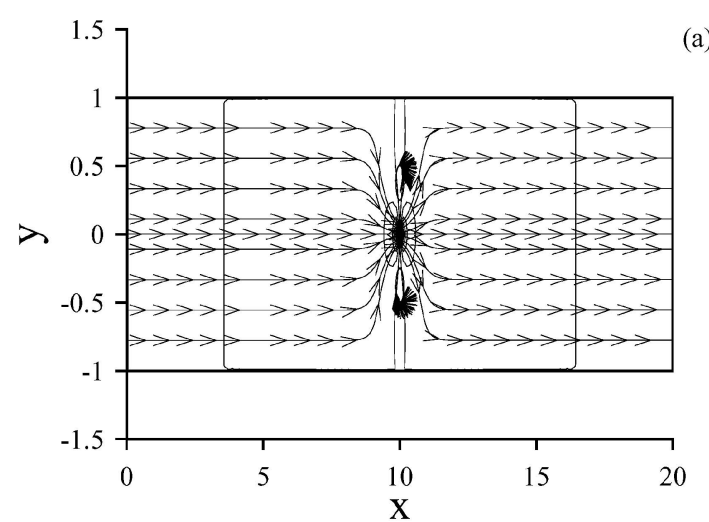

(b)
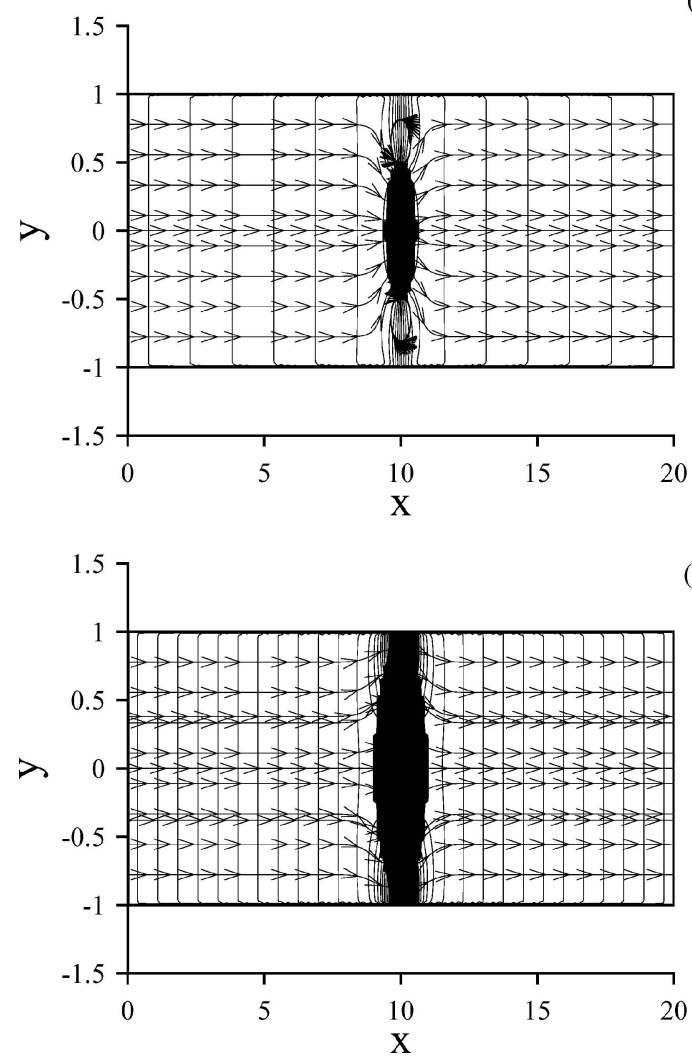

Fig. 7. Streamlines and pressure contours for the straight channel with $L / a=20$ and $\Delta p=0$, for (a) b/a $=0.1,--,(b) b / a=0.5,--$, and (c) $b / a=0.9, \ldots$. The flow is unidirectional far away from the bead, but recirculation occurs near the bead. The pressure is higher in front of the bead than behind. The resulting pressure gradient increases as bead radius is increased, as indicated by the closer spacing of the pressure contours. Note that the pressure contours are constructed in the same manner for each frame, with the range $p= \pm 50$ divided into 15 increments. Fewer pressure contours correspond to a smaller variation in pressure.

as would be expected due to the longer channel length at higher $R_{o} / a=10$.

Figure 9 shows (a) dimensionless force, $F_{x}$, vs. dimensionless outer channel radius, $R_{o} / a$, and (b) dimensionless flow rate, $Q$, vs. dimensionless outer channel radius, $R_{o} / a$
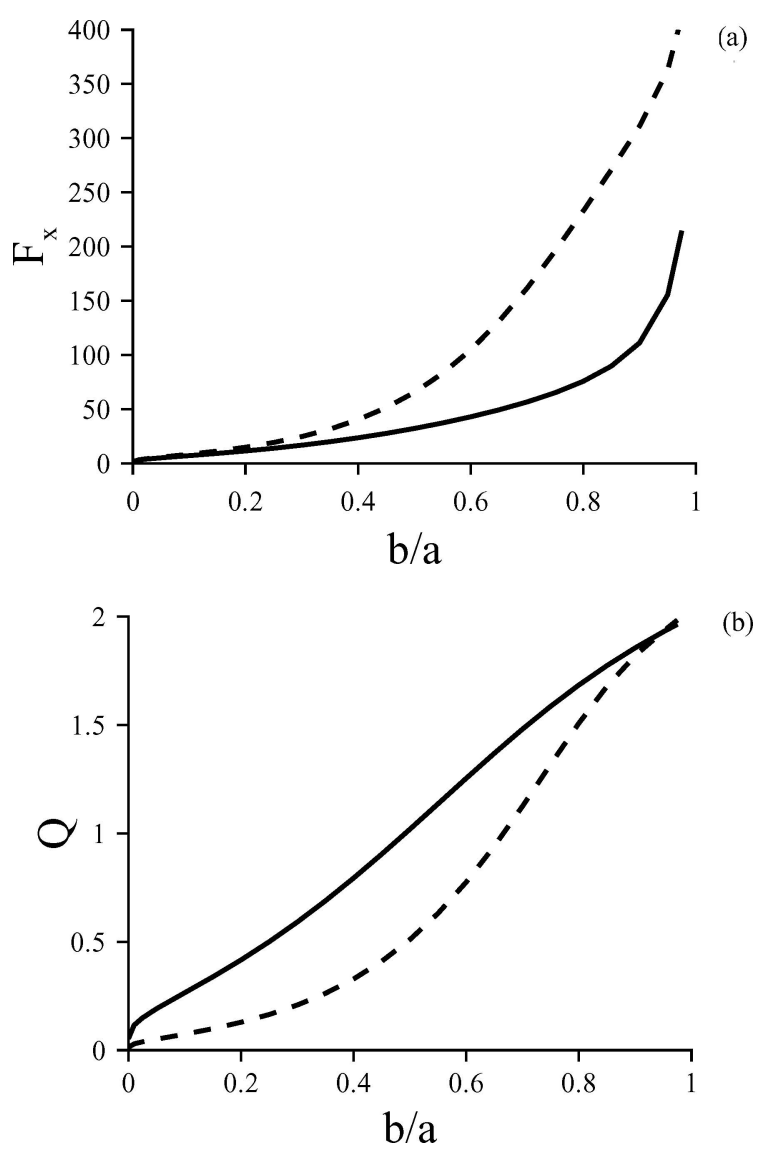

(b)

Fig. 8. (a) Dimensionless force, $F_{x}$, and (b) dimensionless flow rate, $Q$, vs. dimensionless bead radius, $r$, in the circular channel with $R_{o} / a$ $=3,--$, and $(b) R_{o} / a=10$, - -

for $b / a=0.1,0.5$, and 0.9 . As in the straight channel case (Figure 4), the curves are nearly linear for large $R_{o} / a$ and large bead radii, but not for small $R_{o} / a$ and small bead radii, suggesting an interplay between flow near the bead and flow away from the bead in determining the force required to move the bead and the resulting flow rate. This is also indicated by the variation in channel centerline pressure and the wall stresses with radial position, $\theta$. Since circumferential length is given by $\theta \cdot r$ and $r=R_{i} / a+1$ is the centerline radius, $\theta$ in the circular channel geometry is analogous to $x$ in the straight channel geometry. Channel centerline pressure, $p\left(r=R_{i} / a+1\right)$, vs. angular position, $\theta$, for (a) $R_{o} / a=3$ and (b) $R_{o} / a=10$, is shown in Figure 10. Each plot contains lines corresponding to $b / a$ $=0.1,0.5$, and 0.9. In these plots, the center of the bead is located at $\theta=\pi / 2 \approx 1.57$. Near the bead, $p$ is not linear in $\theta$, but away from the bead it is.

Figure 11 shows (a) normal wall stress, $f_{n}$, vs. $\theta$ and (b) tangential wall stress, $f_{\tau}$, vs. $\theta$ for $R_{o} / a=3$; and (c) normal wall stress vs. $\theta$ and (d) tangential wall stress vs. $\theta$ for $R_{o} / a=10$. Each plot contains lines for the stresses on the 

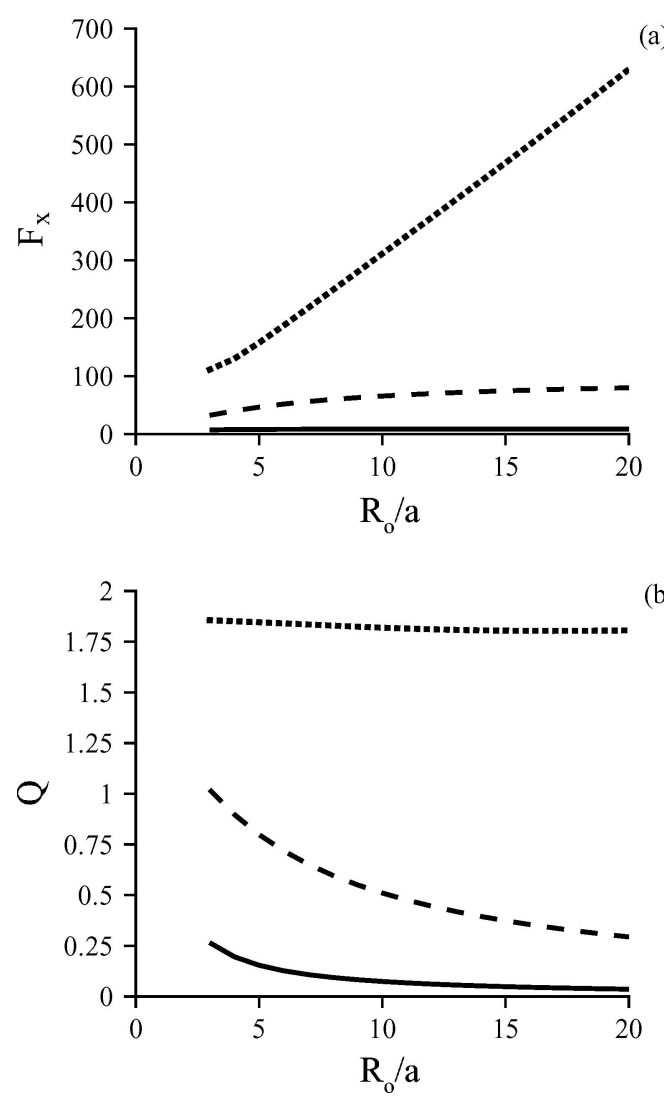

Fig. 9. (a) Dimensionless force, $F_{x}$, vs. dimensionless outer channel radius, $R_{o} / a$, and $(b)$ dimensionless flow rate, $Q$, vs. dimensionless outer channel radius, $R_{o} / a$. Each plot contains lines corresponding to $b / a=0.1,--, b / a=0.5,--$, and $b / a=0.9, \ldots$ These data are from the circular channel model.

outer and inner channel walls for $b / a=0.1,0.5$, and 0.9 . Away from the bead, the stresses vary linearly with $\theta$, but near the bead, there are significant variations. The stresses are higher for higher $b / a$. For each value of $b / a$ the normal stress is higher on the outer wall than on the inner wall when $R_{o} / a=3$. For $R_{o} / a=3$ and $b / a=0.9$, there is an increase (decrease) in the $f_{\tau}$ on the outer (inner) wall near the front and back of the bead, and a decrease (increase) in $f_{\tau}$ near the center of the bead. This indicates the importance of the leakage of fluid around the bead and the corresponding stress field on the required force that must be generated by the kinesin and the resulting flow generated by the pumping mechanism. Note that the different curvature of the inner and outer walls, along with spatial variations in the velocity field, results in different stresses on the inner and outer walls. Similar behavior is noted for $R_{o} / a=10$, except in that case, the differences between stresses on the inner and outer walls are reduced, as the channel curvature is much smaller. Additionally, $R_{o} / a=10$ leads to higher

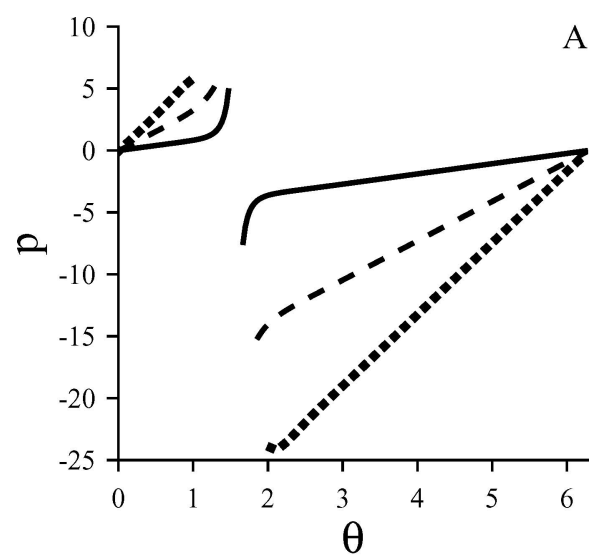

A

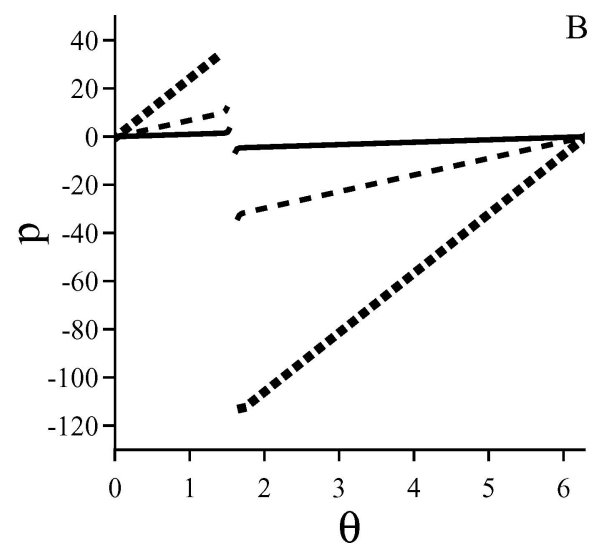

Fig. 10. Channel centerline pressure, $p\left(r=R_{i} / a+1\right)$, vs. angular position, $\theta$, for (a) $R_{o} / a=3$ and ( $b$ ) $R_{o} / a=10$. Each plot contains lines corresponding to $b / a=0.1,--, b / a=0.5,--$, and $b / a=0.9$, ... These data are from the circular channel model. Note there is a jump in pressure at the bead because centerline pressure is undefined in the bead.

pressure gradients and higher normal stresses than does $R_{o} / a=3$.

The effects of bead rotation on the force required to move the bead, $F_{x}$, were considered for the case of the curved channel. Figure 12 shows $F_{x}$ vs. the angular rotation speed of the bead, $\omega$, for a range of bead sizes. Figure 12(a) corresponds to highly curved channel, with $R_{o} / a=$ 3 , and Figure 12(b) corresponds to a less curved channel, with $R_{o} / a=10$. The effects are more pronounced in the higher curvature case, Figure 12(a), but are also noticeable in the less curved channel. Clockwise rotation of the bead reduces the forces required to move the bead. In this motion, the bead appears to roll along the inner wall of the channel. For small beads, the rotation has little effect, but for larger values of $b / a$ the effect is significant $(\sim 200 \%$ change in $F_{x}$ ). The effects of this rotation on flow rate are small $(<2 \%)$ at each value of $b / a$, and thus $Q$ vs. $\omega$ plots are not shown. To understand the mechanisms by which bead rotation effects $F_{x}$, streamlines and pressure fields 

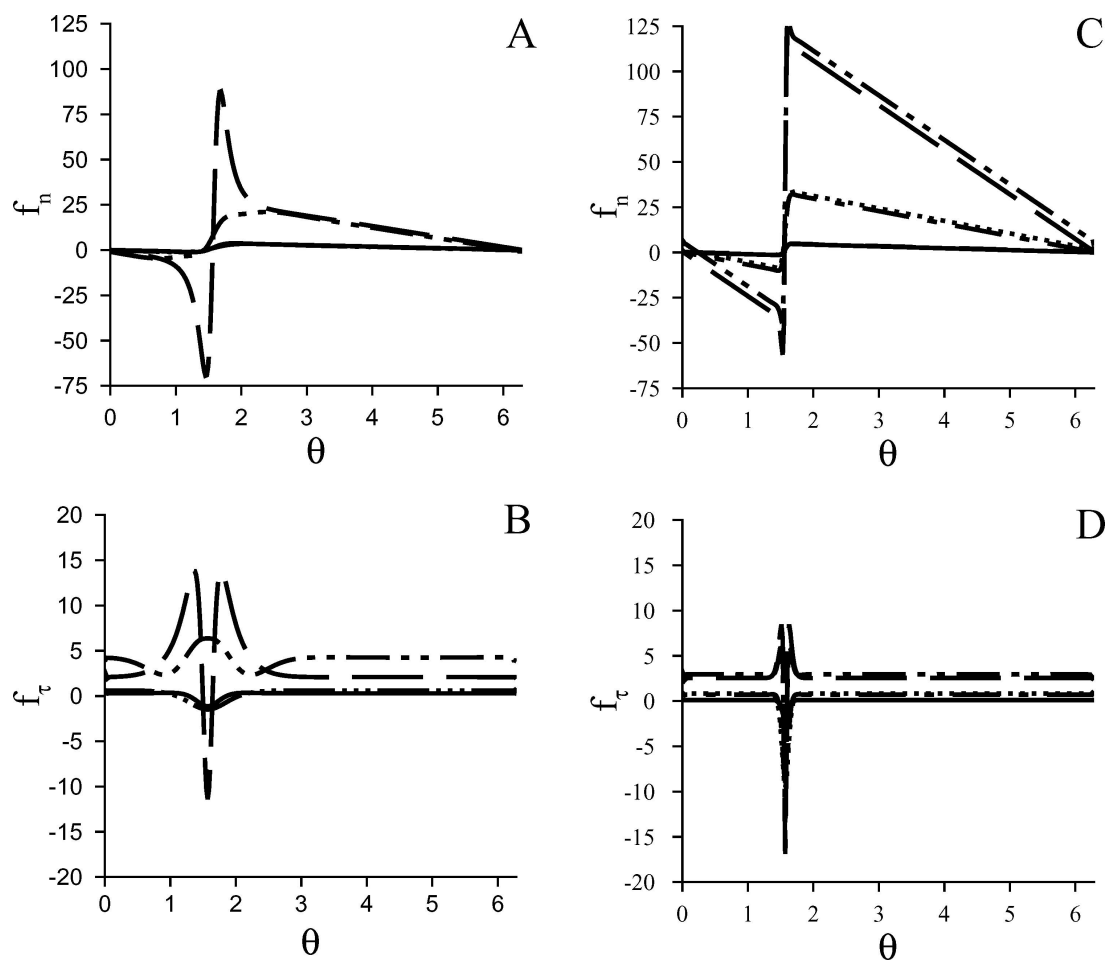

B

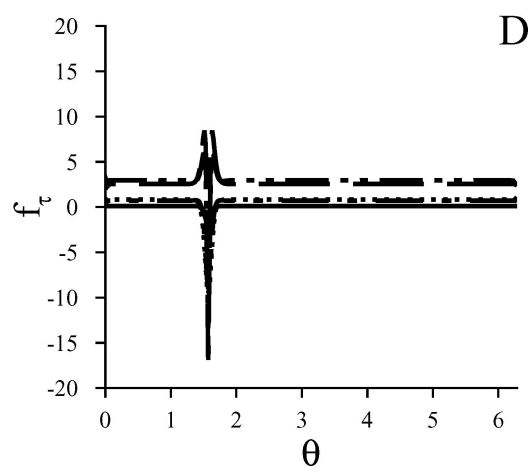

Fig. 11. (a) Normal wall stress vs. $\theta$ and (b) tangential wall stress vs. $\theta$ for $R_{o} / a=3$. (c) Normal wall stress vs. $\theta$ and (d) tangential wall stress vs. $\theta$ for $R_{o} / a=10$. Each plot shows: outer wall stress $b / a=0.1--$, inner wall stressb/a $=0.1,--$, outer wall stress b/a $=0.5-\cdot$, , inner wall stress b/a $=0.5 \ldots$ outer wall stress b/a $=0.9----$, and inner wall stress $b / a=0.9-\cdots-$
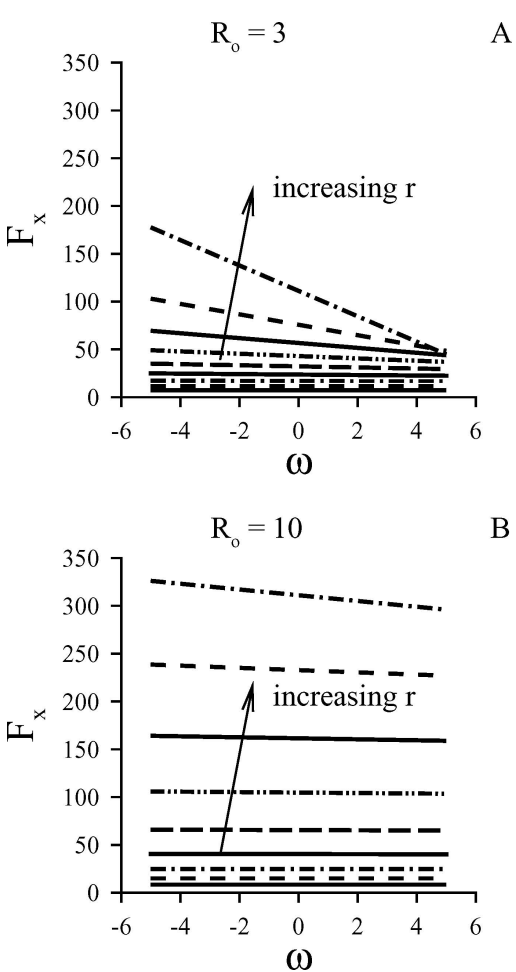

Fig. 12. Bead force, $F_{x}$, vs. rotation frequency, $\omega$, in the circular channel for (a) $R_{o} / a=3$ and (b) $R_{o} / a=10$. were plotted with and without bead rotation, for $R_{o} / a=3$ and several values of $b / a$, as shown in Figure 13. Figure 13(a)-(c) correspond to $b / a=0.1,0.5$, and 0.9 , respectively, with $\omega=0$. Figure 13(d)-(f) correspond to $b / a=$ $0.1,0.5$, and 0.9 , respectively, for $\omega=4$. When the bead does not rotate about its center, regions of recirculation regions form between the bead and the channel walls, as shown in Figure 13(a)-(c). For larger beads, clockwise rotation eliminates the recirculation region between the bead and the channel walls (Figure 13(e) and (f)). Instead, there is some flow in the direction of the bead motion between the bead and the outer wall (at $R_{o} / a$ ) and opposite flow between the bead and the inner wall (at $R_{i} / a$ ). For $b / a=0.1$, the recirculation region remains with bead rotation. For all three bead radii, the clockwise bead rotation reduces the size of the high pressure region in front of the bead (Figure 13), and, therefore, reduces the net force required to move the bead, as indicated in Figure 12.

Plots of dimensionless force, $F_{x}$, and dimensionless flow rate, $Q$, vs. dimensionless bead shift, $Y / a$, are shown in Figure 14 for $b / a=0.5$, and $R_{o} / a=3$ and $10 . Q$ is lower for $R_{o} / a=10$ than for $R_{o} / a=3$ because the viscous losses are higher due to the longer channel. Likewise, the $F_{x}$ is higher for $R_{o} / a=10$, except when the bead is positioned very close to the channel wall. Shifting the bead slightly from the centerline of the channel towards the 

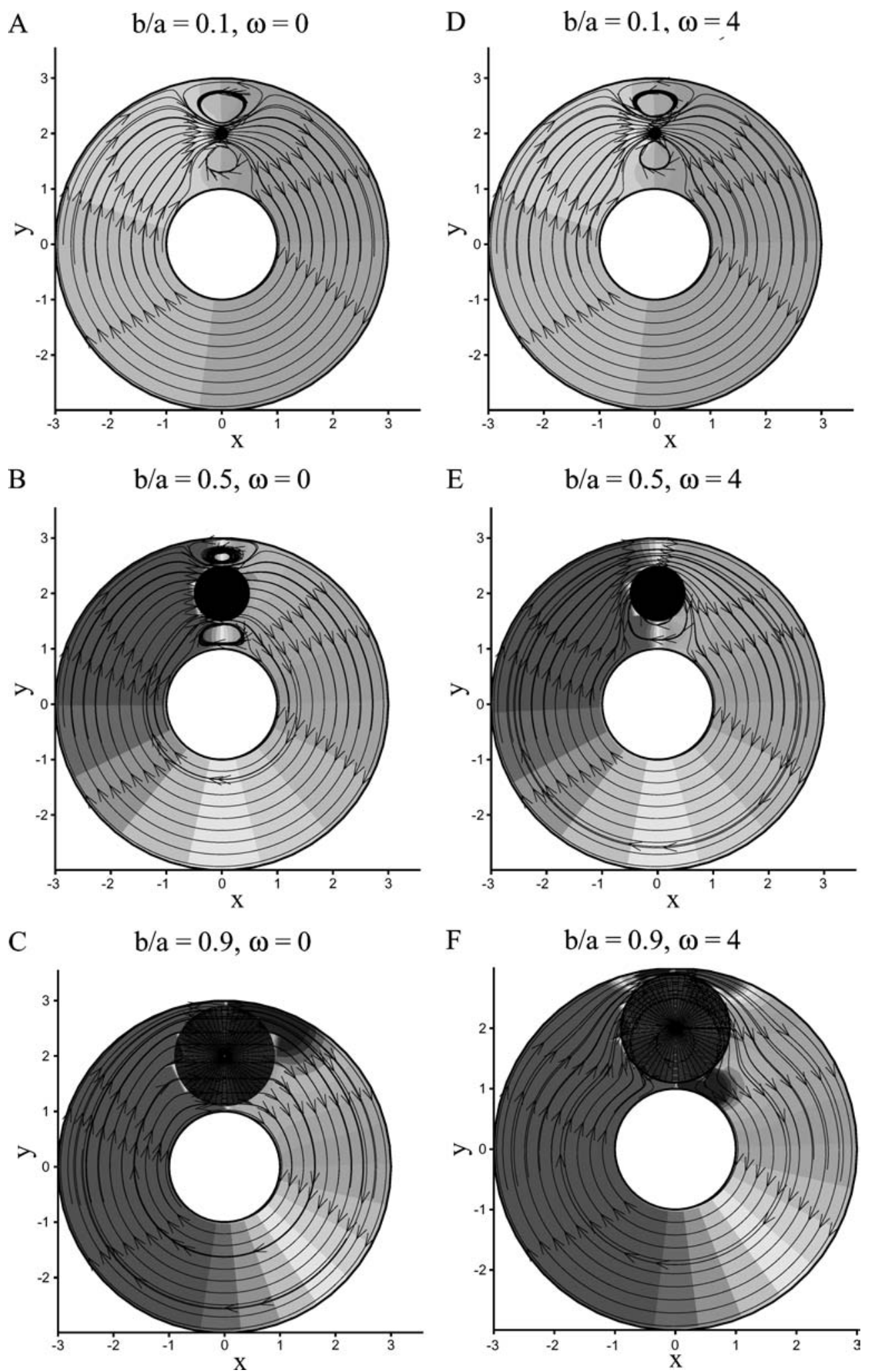

Fig. 13. Streamlines and pressure contours for the circular channel with $\omega=0$ when (a) $b / a=0.1,(b) b / a=0.5,(c) b / a=0.9$, and $\omega=4$ when (c) $b / a=0.1,(d) b / a=0.5,(e) b / a=0.9$. The streamlines indicate recirculation near the bead, and flow parallel to the channel walls away from the bead. Note that the contour levels for the pressure field range from -10 to 10 (dimensionlessp) and there are 15 levels in each plot. The highest pressures occur in front of the bead and the lowest behind the bead. 


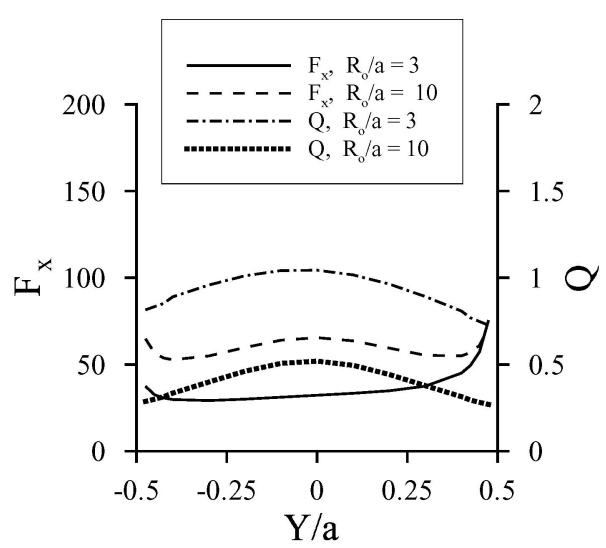

Fig. 14. Dimensionless force, $F_{x}$, and dimensionless flow rate, $Q$, vs. dimensionless bead shift, $Y / a$, in the circular channel with $b / a=0.5$, and $R_{o} / a=3$ and 10 .

inner wall results in a decrease in $F_{x}$ and an increase in $Q$. The pressure and velocity fields are not symmetric about the channel centerline, as shown in Figure 13(b), because of channel curvature. Shifting the bead slightly inward reduces the effect of the outer wall, which results in higher pressures near the outer wall in the vicinity of the bead. Given the high accuracy with which proteins can be patterned (Hoff et al., 2004), this shifting of the bead slightly inward is potentially an achievable design modification.

\subsection{Limitations}

This model is an idealization of the flow induced by kinesin-powered micro-bead motion, and there are a number of simplifications and limitations. The model is twodimensional, and ignores details of the top and bottom walls of the channel. Although two-dimensional models have been successful in capturing the behavior of corresponding three-dimensional situations (Pozrikidis, 1997b; Gaver and Kute, 1998), the model here may underestimate viscosity effects due to the neglect of the top and bottom walls of the channel. Additionally, we have not modeled the connection of this device to an external circuit, which likely increases the losses in the system. Therefore the efficiencies and flow rates predicted by this model are likely higher than would be expected in the actual situation. Nevertheless, this model provides an indication that this micropump design is feasible and provides guidance for its optimization. Efficient and adaptable models such as this one will be a powerful tool in designing biomolecular-motor-powered-microfluidics devices. Future work should include valves and external connections, should account for the effects of load on the kinesin speed, and should consider transport of ATP to fuel the kinesin. Further investigation and refinement of the model will lead to a better understanding of the transport phenomena in the micropump, and subsequently an optimized micropump.

\section{Conclusions}

The theoretical study presented here demonstrates the boundary element method is a powerful tool for the analysis and design of microfluidic devices. The model results suggest that our novel microfluidics pump will be relatively efficient and can drive a substantial external load while delivering flows in the atto- to pico-1/s range. The flows generated by the pump increase with bead radius. The force required to move the bead also increases with bead radius, but at a slower rate than the flow rate increases until the bead nearly occludes the channel, indicating that large beads are preferred from the stand point of generating maximum flows. The efficiency of the pump increases with bead size and larger bead sizes are able to support a higher imposed pressure. Bead rotation in the same direction as bead translation in the circular channel model reduces the force required to move the bead with minimal effect on flow rate. Shifting the bead slightly inward also reduces the force required to move the bead. This initial theoretical model suggests that this micropump design may provide an efficient alternative to existing micropump technologies, and provides a starting point for optimization of the current pump design and investigation of more complicated pump geometries.

\section{Acknowledgments}

This work was supported by DARPA BAA01-47.

\section{References}

D.A. Ateya, A.A. Shah, and S.Z. Hua, Review of Scientific Instruments 75, 915-920 (2004).

C.A. Brebbia and J. Dominguez, Boundary Elements-An Introductory Course (Southampton, Computational Mechanics, 1992), $313 \mathrm{p}$.

F.P. Bretherton, Journal of Fluid Mechanics 14, 284-304 (1962).

S. Childress, Mechanics of Swimming and Flying, (Cambridge University Press, 1981).

E.R. Damiano, B.R. Duling, K. Ley, and T.C. Skalak, Journal of Fluid Mechanics 314, 163-89 (1996).

E.R. Damiano, D.S. Long, F.H. El-Khatib, and T.M. Stace, Journal of Fluid Mechanics 500, 75-101 (2004).

A.M.J. Davis, Physics of Fluids a-Fluid Dynamics 4, 7-15 (1992).

W.R. Dean and M.E. O’Neill, Mathematika 10, 13-24 (1963).

P.W.T. Dierickx, D. De Wachter, and P.R. Verdonck, International Journal of Artificial Organs 23, 610-617 (2000).

A.S. Dvinsky and A.S. Popel, Computers and Fluids 15, 391-404 (1987a).

A.S. Dvinsky and A.S. Popel, Computers and Fluids 15, 405-419 (1987b).

E. Eklund and A. Jernqvist, Chemical Engineering Science 49, 37653772 (1994).

J. Feng, P. Ganatos, and S. Weinbaum, Journal of Fluid Mechanics 375, 265-296 (1998). 
P. Ganatos, R. Pfeffer, and S. Weinbaum, Journal of Fluid Mechanics 84, 79-111 (1978).

P. Ganatos, R. Pfeffer, and S. Weinbaum, Journal of Fluid Mechanics 99, 755-783 (1980a).

P. Ganatos, S. Weinbaum, and R. Pfeffer, Journal of Fluid Mechanics 99, 739-753 (1980b).

P. Ganatos, S. Weinbaum, and R. Pfeffer, Journal of Fluid Mechanics 124, 27-43 (1982).

D.P. Gaver and S.M. Kute, Biophysical Journal 75, 721-733 (1998).

X. Geng, H. Yuan, H.N. Oguz, and A. Prosperetti, Journal of Micromechanics and Microengineering 11, 270-276 (2001).

A.J. Goldman, R.G. Cox, and H. Brenner, Chemical Engineering Science 22, 653 (1967a).

A.J. Goldman, R.G. Cox, and H. Brenner, Chemical Engineering Science 22, 637 (1967b).

S.L. Goren and M.E. Oneill, Chemical Engineering Science 26, 325 (1971).

D. Halpern and T.W. Secomb, Journal of Fluid Mechanics 231, 545-560 (1991).

D. Halpern and T.W. Secomb, Journal of Fluid Mechanics 244, 307-322 (1992).

J. Happel and H. Brenner, Low Reynolds Number Hydrodynamics. (Prentice-Hall, Englewood Cliffs, N.J., 1965) p. 553.

E.F. Hasselbrink, T.J. Shepodd, and J.E. Rehm, Analytical Chemistry 74, 4913-4918 (2002).

H. Hess, G.D. Bachand, and V. Vogel, Chemistry-a European Journal 10, 2110-2116 (2004).

N. Hirokawa, Science 279, 519-526 (1998).

J.D. Hoff, L.J. Cheng, E. Meyhofer, L.J. Guo, and A.J. Hunt, Nano Letters 4, 853-857 (2004).

J. Howard, Annual Review of Physiology 58, 703-729 (1996).

A.J. Hunt, F. Gittes, and J. Howard, Biophysical Journal 67, 766-781 (1994).

L.L. Jia, S.G. Moorjani, T.N. Jackson, and W.O. Hancock, Biomedical Microdevices 6, 67-74 (2004).

B.J. Kirby, T.J. Shepodd, and E.F. Hasselbrink, Journal of Chromatography A 979, 147-154 (2002).

O.A. Ladyzhenskaya, The Mathematical Theory of Viscous Incompressible Flow, (Gordon and Breach New York, 1963).
R.E. Larson and J.J.L. Higdon, Journal of Fluid Mechanics 166, 449-472 (1986).

R.E. Larson and J.J.L. Higdon, Journal of Fluid Mechanics 178, 119-136 (1987).

Z.X. Li, M.R. Wang, X.B. Yao, and Z.Y. Guo, Microscale Thermophysical Engineering 8, 31-41 (2004).

L. Limberis and R.J. Stewart, Nanotechnology 11, 47-51 (2000).

H. Liu, H.H. Bau, and H. Hu, Physics of Fluids 16, 998-1007 (2004).

E. Meyhofer and J. Howard, Proceedings of the National Academy of Sciences of the United States of America 92, 574-578 (1995).

J.Y. Min, E.F. Hasselbrink, and S.J. Kim, Sensors and Actuators BChemical 98, 368-377 (2004).

S.G. Moorjani, L. Jia, T.N. Jackson, and W.O. Hancock, Nano Letters 3, 633-637 (2003).

M.E. O’Neill, Mathematika 11, 67-74 (1964).

E. Ory, H. Yuan, A. Prosperetti, S. Popinet, and S. Zaleski, Physics of Fluids 12, 1268-1277 (2000).

C. Pozrikidis, Boundary Integral and Singularity Methods for Linearized Viscous Flow, (Cambridge University Press, 1992), p. 259.

C. Pozrikidis, Journal of Fluid Mechanics 261, 199-222 (1994).

C. Pozrikidis, Introduction to Theoretical and Computational Fluid Dynamics, (Oxford University Press, Oxford, 1997a) p. 675.

C. Pozrikidis, Journal of Engineering Mathematics 31, 29-42 (1997b).

E.M. Sparrow and A.L. Loeffler, A.I.Ch.E. Journal 5, 325-330 (1959).

M.E. Staben, A.Z. Zinchenko, and R.H. Davis, Physics of Fluids 15, 1711-1733 (2003).

M. SugiharaSeki, Journal of Fluid Mechanics 324, 287-308 (1996).

M. Sugiharaseki and R. Skalak, Microvascular Research 36, 64-74 (1988).

S.P. Sutera and R. Skalak, Annual Review of Fluid Mechanics 25, 1-19 (1993).

D.B. Thiessen and W.B. Krantz, Review of Scientific Instruments 63 , 4200-4204 (1992).

C.Y. Wang, International Journal of Multiphase Flow 22, 185-194 (1996).

E.G. Wehbeh, T.J. Ui, and R.G. Hussey, Physics of Fluids a-Fluid Dynamics 5, 25-33 (1993).

H. Yuan and A. Prosperetti, Journal of Micromechanics and Microengineering 9, 402-413 (1999). 